\title{
La Simulación como Medio de Interrelación entre Herramientas Matemáticas y Procesos Tecnológicos
}

\author{
Griselda I. Cistac, Ruben H. Bongianino, José L. Filippi y Federico D. Kovac \\ Universidad Nacional de La Pampa, Facultad de Ingeniería, Calle 9 esquina 110, General Pico, \\ La Pampa-Argentina (e-mail: cistacg@ing.unlpam.edu.ar; bongianino@ing.unlpam.edu.ar)
}

\begin{abstract}
Resumen
El objetivo del trabajo es lograr una articulación vertical entre asignaturas básicas y tecnológicas en los primeros años de la carrera de ingeniería, de tal forma que los estudiantes dejen de ver a las mismas como compartimientos estancos, relacionando la matemática con áreas tecnológicas utilizando un software de simulación. La actividad en aula involucra docentes de distintas asignaturas, quienes conjuntamente presentan un sistema físico que debe ser modelado, resuelto mediante Transformada de Laplace y simulado. Una encuesta en sala muestra que la propuesta resulta de utilidad, y contribuye a la comprensión e internalización entre el concepto matemático y su aplicación. Se concluye que la experiencia logra internalizar e integrar conceptos matemáticos y tecnológicos. Además, la simulación permite visualizar respuestas y variar parámetros realizando un análisis crítico, actividades que despiertan interés en los estudiantes y motivan su proceso de aprendizaje.
\end{abstract}

Palabras clave: simulación, interrelación, procesos tecnológicos, herramientas matemáticas, estudiantes

\section{Simulation as Interrelationship between Mathematic Tools and Technological Process}

\begin{abstract}
The object of this work is to achieve a vertical interrelationship between basic science and technological subjects in the initial courses of engineering so that students, through simulation software, start relating both areas instead of considering them unrelated fields. The activity involves teachers of various courses who define a physical situation to be modelled, solved using Laplace Transform, and then simulated. A class survey shows that the proposal is useful and contributes to the understanding and internalization of the mathematical concept and its application. It is concluded that the experience does internalize and integrate mathematical and technological concepts. Also, the simulation allows visualizing the answers and varying the process parameters to do a critical analysis, activities that attract student attention and motivate their learning process.
\end{abstract}

Keywords: simulation, interrelationship, technological process, mathematic tools, students 


\section{INTRODUCCION}

La separación temporal existente en los actuales planes de estudio de las Carreras de Ingeniería en general, hace que cuando los estudiantes adquieren los conceptos matemáticos no tienen los conocimientos tecnológicos necesarios para aplicarlos a problemas físicos, y cuando estudian los procesos en las tecnológicas, las herramientas matemáticas están 'un poco olvidadas' .La disociación que parece plantearse entre los conceptos matemáticos y los procesos tecnológicos hace que los estudiantes vean a las distintas áreas como compartimientos estancos, no logrando una interrelación interesante entre asignaturas básicas con tecnológicas. Estudios realizados manifiestan que “...Entender el significado de la integración de contenidos en el currículo disciplinar puede ser la vía para proponer algunas actividades que se pueden implementar para lograr este propósito. ..." (Vega, 2003). La disociación o falta de interrelación de contenidos da lugar a que éstos queden anclados dentro de la asignatura, idea que refuerza (Ojeda y Alcalá, 2007) cuando mencionan "...si bien existe un marcado anclaje disciplinar, aparecen componentes - en mayor o menor medida - de la práctica profesional. También, es posible señalar como particularidad de algunas cátedras, el interés de habilidades y estrategias de aprendizaje y estudio para favorecer la ambientación y el rendimiento de los alumnos que ingresan a un ámbito educativo con características específicas." En la etapa inicial de la carrera una primera aproximación hacia la práctica profesional es el contacto con las asignaturas tecnológicas básicas.

En los primeros años de la carrera esta interrelación resulta difícil fundamentalmente entre Matemática, Física y algunas Tecnologías Básicas por parte de los estudiantes ya que están en la construcción inicial de sus capacidades técnicas. En general no pueden lograrla, porque cuando en matemática les enseñan los conocimientos propios resuelven ecuaciones (no asociadas a un proceso físico) y cuando en las tecnológicas parten de un proceso físico el que deben modelar (hallar la ecuación) no tienen totalmente internalizados los conceptos matemáticos. Cuando se menciona interrelacionar contenidos, no es que necesariamente el $100 \%$ de los mismos deba o pueda lograrse, lo que se marca puntualmente es que en todo proceso inicial de una Carrera de Ingeniería la interrelación es FÍSICA-MATEMÁTICA-TECNÓLOGICAS BÁSICAS ya que en esta trilogía, la Física aporta las conceptualizaciones necesarias para entender un fenómeno en particular, la Matemática permite el modelado del mismo y los procesos resolutivos, y las Tecnológicas aplican estos resultados para producir productos o procesos, para lo cual es necesario tener presente la siguiente secuencia: problema físico $\Rightarrow$ planteo matemático $\Rightarrow$ solución del problema

Una alternativa válida para subsanar los inconvenientes marcados, es la planteada en el marco del proyecto de investigación ("Integración de conceptos de Análisis III, Electrotecnia y Teoría de Control I mediante software de simulación partiendo de sistemas físicos de interés particular") vigente en la Facultad de Ingeniería de la Universidad Nacional de La Pampa donde se proponen actividades áulicas a través de una metodología distinta (a las utilizadas habitualmente) de modo de lograr una interacción vertical entre Análisis III (Transformada de Laplace), con Electrotecnia General (circuitos eléctricos), y con Teoría de Control I (diagrama de bloques y el software específico de simulación). Al tomar la interacción como una posible solución a la situación planteada las preguntas que surgen son si la misma la deben realizar ¿los docentes en sus cátedras?, ¿los estudiantes al construir sus propios aprendizajes? o ¿es una tarea conjunta entre las distintas cátedras?.

Las dos primeras preguntas no aportan significativamente ni a la integración ni a la internalización, ya que en la práctica el profesor no siempre puede desarrollar un proceso de enseñanzaaprendizaje que facilite al estudiante ver el contenido como un todo (Ruiz, 2008). La tercera es respondida en parte por la postura sustentada por (Vega, 2003) cuando afirma que: "la interdisciplinariedad implica el encuentro y la cooperación entre dos o más disciplinas, cada una de ellas contribuyendo, en un nivel teórico o de investigación empírica con sus esquemas conceptuales propios, su manera de definir los problemas y sus métodos de análisis. Un enfoque interdisciplinar exige que se trabaje con una misma tarea científica o una determinada situación de aprendizaje desde diferentes ángulos". La respuesta dada desde el proyecto concuerda con la posición de (Vega, 2003), planteando actividades áulicas entre Análisis III, Electrotecnia General, y Teoría de Control I, involucrando a los docentes de esas cátedras quienes realizan una actividad 
compartida en forma conjunta (brindando con distintos enfoques la misma información), para ayudar a los estudiantes en un proceso que les permita 'lograr la conexión de los conocimientos de las distintas asignaturas de modo que dejen de verlas como compartimientos estancos'. Esta tarea conjunta puede pensarse como una estrategia de intervención docente (Gimeno y Pérez, 1997) para modificar el proceso de enseñanza-aprendizaje tratando de evitar la mecanización de los contenidos que no permiten la internalización ni la interrelación de los mismos. Las actividades mencionadas tienen como objetivo que los estudiantes al término de las mismas sean capaces de lograr en forma más rápida entender que los conocimientos matemáticos sirven para resolver problemas tecnológicos y que no son un fin en si mismos, internalizar las herramientas matemáticas relacionándolas con situaciones de las áreas tecnológicas acorde a los conocimientos previos. También se tenderá en lo posible a la articulación vertical entre asignaturas básicas y tecnológicas con una adecuada interrelación teórico-práctica tomando exacta dimensión del problema físico-planteo matemático-solución del problema utilizando para tal fin herramientas virtuales.

La presente propuesta es sustentada por trabajos similares que buscan aprendizajes significativos y estrategias para que los estudiantes se conviertan en aprendices reflexivos (Cid, 2008), propuestas didácticas para la enseñanza de la física (Valdés y Valdés, 1999, Douglas et al. 2006), vinculación de la teoría-práctica de la matemática a través de un enfoque constructivista (Mora, 2005), estrategias integradoras en la enseñanza (Vásquez et al., 2004), vinculación de la matemática con contenidos de la realidad (Ruiz, 2008) que muestran la preocupación y el interés existente en el ámbito educativo buscando alternativas para evitar que las distintas áreas actúen como compartimientos que no interactúan entre sí.

Esta preocupación para mejorar el proceso de enseñanza-aprendizaje es investigada en un marco general desde la epistemología de la ciencia, y por otros desde su área disciplinar. El trabajo presentado se sustenta en la integración parcial de un contenido específico de matemática (Transformada de Laplace) con circuitos eléctricos lineales (Resistencias, Capacitores, Inductancias) y su correspondiente análisis mediante herramientas virtuales (software de simulación). El concepto matemático aunque pueda interpretarse como puntual en esta instancia de la carrera, es de alcance mucho más amplio ya que se retoma en Teoría de Control I (Tecnológica Aplicada) y a partir de él se desarrollan los conceptos propios de la disciplina del Control Clásico. El ir adquiriendo el conocimiento y manejo del software de simulación en esta etapa familiariza a los estudiantes con la herramienta, de modo que cuando la utilicen en Teoría de Control I para el análisis y diseño de estructuras de control automático la misma no es totalmente nueva.

\section{METODOLOGIA}

El proceso enseñanza-aprendizaje de los conocimientos de las Ciencias Básicas (Matemática, Física) ha sido analizado por distintos autores desde proyectos de investigación o de aplicación en el aula, involucrando resolución de problemas, integración teoría y práctica, apropiación del lenguaje simbólico, integración de contenidos de la ciencia (Santos, 1997; Nieto, 2005; Douglas et al., 2006; Linn et al. 2006; Santos, 2008) que muestran un camino posible para integrar contenidos y lograr aprendizajes significativos. En esta misma línea, y a través de una propuesta diferente a las utilizadas generalmente en nuestra Facultad, se llevan a cabo actividades áulicas que en forma conjunta los docentes de las distintas áreas presentan Transformada de Laplace, Sistema Mecánico de Traslación, Circuitos Eléctricos, Diagramas de Bloques y de Simulación. El tema de Transformada de Laplace, que permite entre una de sus posibilidades resolver ecuaciones diferenciales (Análisis III) es el seleccionado para analizar y modelar: los circuitos eléctricos lineales (Electrotecnia General) y el sistema mecánico de traslación. El diagrama de bloques es el que permitirá crear el de simulación para analizar y visualizar los resultados (Teoría de Control I).

Para lograr la vinculación de los temas mencionados el dictado de los mismos se lleva a cabo en las clases de Análisis III y Electrotecnia General. En Análisis III antes de la propuesta se aplicaban los conocimientos de Transformada de Laplace a una ecuación diferencial genérica sin estar asociada a un proceso físico que lo modelara. Esta propuesta plantea como punto de partida el análisis de un proceso físico (el que puede ser desarrollado con los conocimientos de los estudiantes que se hallan cursando Segundo Año) el cual debe estar modelado (por las ecuaciones dinámicas adecuadas) de modo de poder aplicar la Transformada de Laplace para 
resolver y analizar las ecuaciones diferenciales del proceso. "Gravemeijer y Doorman (1999), Lesh y Zowojewski (2007) mencionan:" ... la relevancia de considerar situaciones auténticas o realistas propician en los estudiantes, la construcción de modelos matemáticos (Santos, 2008)". Para adecuar tal situación según los conocimientos previos de los estudiantes el sistema físico bajo análisis es uno mecánico de traslación (formado por una masa, resorte y amortiguador al que se le aplica una fuerza constante) para luego analizar el desplazamiento, velocidad y aceleración que adquieren los distintos componentes. Una vez modelado el sistema y resuelto utilizando Transformada de Laplace (evidenciando su potencialidad frente a otras herramientas matemáticas) se implementa un diagrama de simulación de modo de visualizar gráficamente las soluciones analíticas halladas anteriormente. La simulación es el puente que permitirá el análisis y una mejor interpretación de los resultados, puesto que la visualización ayuda a internalizarlos mejor. En Electrotecnia General los estudiantes se hallan cursando Tercer Año, anteriormente los problemas físicos específicos de la disciplina eran analizados según los procedimientos resolutivos que se encuentran en la mayoría bibliografía (como por ejemplo resolución de ecuaciones diferenciales mediante variables separables, etc.) para obtener el comportamiento de los mismos. En este caso la ecuación matemática que los modela se resuelve utilizando Transformada de Laplace y al igual que en Análisis III se muestra su potencialidad al analizar conjuntamente las respuestas transitoria y permanente. Los problemas físicos bajo estudio son circuitos eléctricos conformados por R (Resistencia) L (Inductancia) C (Capacitor) alimentados con tensión continua o alterna a los efectos de analizar los valores de I (Corriente) que circula por cada elemento y de $\mathrm{V}$ (Tensión) aplicados a cada uno de ellos. Al igual que en Análisis III se utiliza la simulación como herramienta que permite corroborar o no el análisis teórico. En caso de no existir concordancia entre lo resuelto y lo simulado se deben analizar las posibles fuentes de error, es decir el estudiante para hallarlas puede hacerse las siguientes preguntas: ¿Se plantearon mal las ecuaciones?, ¿Está mal implementada la simulación?, ¿Cuál es el origen del error? Las respuestas a estos interrogantes son parte del proceso de aprendizaje donde a partir de errores o resultados no satisfactorios se-replantea la situación lográndose así un análisis crítico.

Cuando los estudiantes realizan una simulación, previamente han desarrollado el modelo matemático del proceso físico, el que debe ser implementado en el software (utilizado en la disciplina de control) lo que hace que la simulación no sea un proceso solamente demostrativo. Otra característica importante para el análisis de procesos físicos mediante la simulación es la facilidad para variar parámetros (de modo de poder analizar los distintos tipos de respuesta de los sistemas). Su incorporación es resaltada como un "facilitador del aprendizaje, un medio de ayuda al profesor..." (Valdés y Valdés, 1999) y como una herramienta que brinda la posibilidad de visualizar problemas científicos complejos como así también ver críticamente el tema (Linn et al., 2006). Como complemento de la propuesta áulica integradora se ha desarrollado material multimedial donde los alumnos encuentran conceptos teóricos, y ejercitación que les permite realizar la autoevaluación de sus conocimientos. Los temas desarrollados son los directamente relacionados con la actividad planteada: Transformada de Laplace, Circuitos eléctricos, Diagrama de bloques (Cistac et al., 2007; 2008a, 2008b). La Figura 1 muestra un esquema que sintetiza la actividad áulica que se desarrolla en cada asignatura.

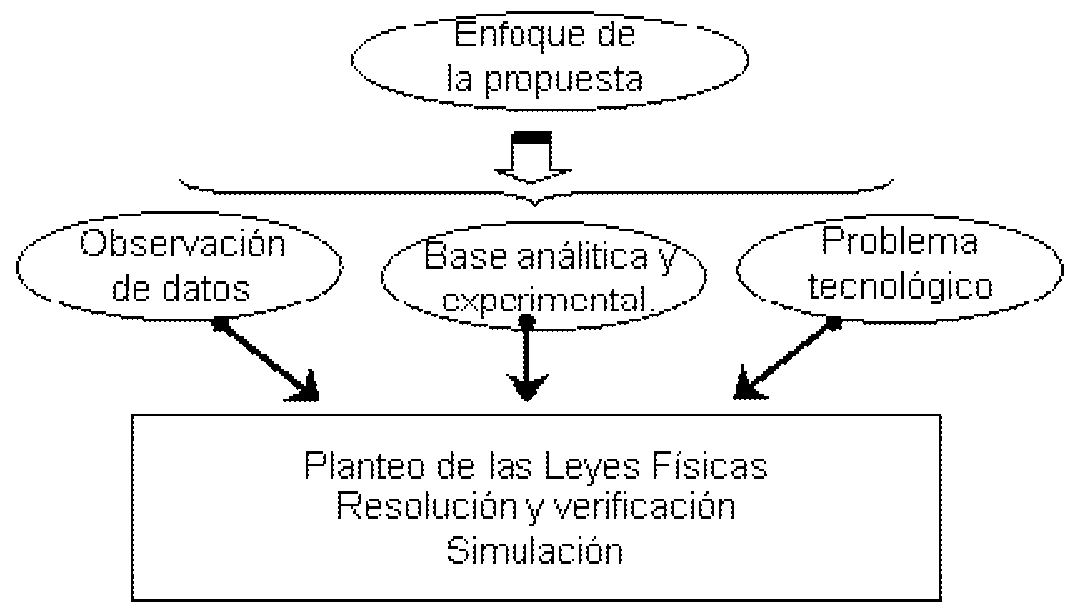

Fig.1: Esquema de la propuesta 
El universo sobre el cual se realiza la recolección de datos está formado por el total de alumnos que cursan cada una de las asignaturas involucradas. Durante un año académico existe solo un curso por asignatura a lo largo de la carrera (no existiendo cursos paralelos) ya que la Institución pertenece al grupo denominado 'Facultades pequeñas'. Para analizar y obtener las conclusiones de las experiencias áulicas tanto en Análisis III como en Electrotecnia General, se utiliza como instrumento una encuesta de diseño propio, en lugar de adoptar alguno de los existentes. La misma está estructurada de modo tal que permite extraer conclusiones relacionadas con el aporte de la actividad en el proceso de enseñanza aprendizaje, la necesidad de reformulaciones de la actividad propiamente dicha o del instrumento de evaluación y el grado de satisfacción de los estudiantes. Durante el desarrollo de las actividades los docentes se encuentran en estrecha relación con los estudiantes debido a la baja relación docente/alumno permitiendo desde lo actitudinal ir percibiendo las frustraciones y logros que son registrados.

\section{RESULTADOS Y DISCUSIÓN}

Los datos obtenidos de las encuestas en el aula tabulados y procesados transformados en tablas y figuras, permiten luego de ser analizados obtener conclusiones sobre los aportes de la metodología y la actividad al proceso de enseñanza aprendizaje. No se evidencia en esta etapa de análisis las modificaciones que se deben realizar al instrumento cuando algunos de los ítems requieren su reformulación. La misma puede presentarse en la carga de datos cuando por ejemplo la respuesta dada por el estudiante permite inferir que no estaba clara la consigna, no aporta significativamente al análisis, o surgen nuevos indicadores a incorporar.

Agrupando algunos de los ítems relacionados con la metodología, las preguntas están orientadas de modo de saber si la actividad permite: ¿interpretar mejor los fenómenos físicos estudiados?, ¿interrelacionar mejor los conceptos matemáticos, con los procesos físicos?, ¿verificar en forma más rápida si la resolución matemática es correcta?, ¿se debe replantear la misma?. Algunos de los ítems que involucran a la propia actividad es si la misma posibilita: recordar conceptos de distintas áreas, seleccionar distintas herramientas matemáticas para resolver ecuaciones diferenciales (métodos tradicionales y Transformada de Laplace) y evaluar la potencialidad de cada uno de ellos, determinar que otros temas y que otras asignaturas podrían interrelacionarse con proyectos similares. Los ítems seleccionados para su análisis son algunos de los que se relacionan por un lado con la metodología y por el otro con la actividad propiamente dicha. Los resultados comparativos de los ítems seleccionados de las encuestas se presentan a continuación en las (Tablas 1 a 5 y Figuras de 2 a 6):

Tabla 1: Pregunta extraída de la encuesta áulica.

\begin{tabular}{|l|c|c|}
\hline Habiendo estudiado con anterioridad Transformada de Laplace, & Año & Año \\
\cline { 2 - 3 } con esta experiencia logró interpretar su aplicación a situaciones & 2006 & 2007 \\
\cline { 2 - 3 } específicas y visualizar su potencialidad. & $\%$ & $\%$ \\
\hline 1 En lo absoluto & 0 & 0 \\
2 Un poco más & 92 & 90 \\
3 Mucho más & 8 & 10 \\
\hline
\end{tabular}

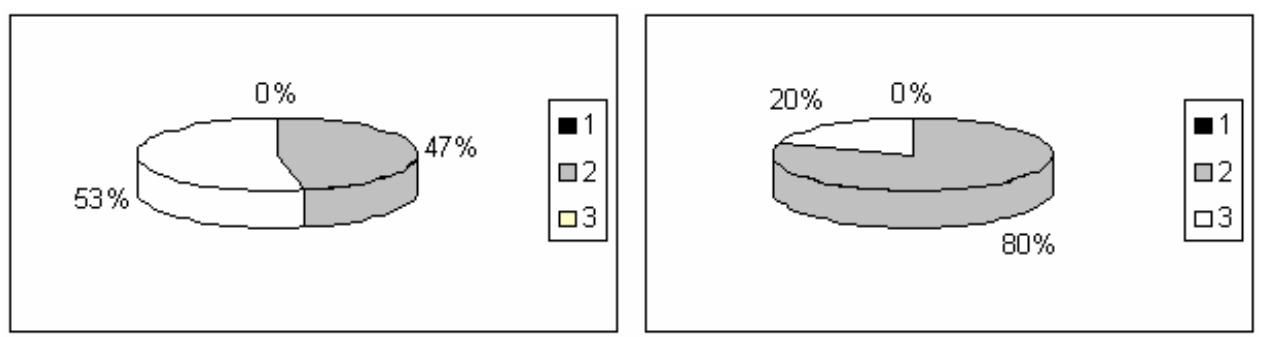

Fig. 2: Lograron interpretar la aplicación de Transformada de Laplace a situaciones específicas Años 2006 y 2007

De acuerdo a los indicadores obtenidos y realizando un análisis comparativo de los mismos surge que han logrado interpretar las aplicaciones matemáticas (Transformada de Laplace) a situaciones específicas en un gran porcentaje, ya que en los dos años $(2006,2007)$ alrededor del $90 \%$ de los 
estudiantes manifiestan haberlo interpretado un poco más, un $10 \%$ mucho más y ningún estudiante en lo absoluto (Tabla 1). Con relación a la vinculación de los contenidos de matemática con la aplicación al caso en estudio, se destaca que para todos los estudiantes la misma se logró, y ninguno indica no haberlo conseguido (Tabla 2). Estos datos en conjunto sirven como indicadores de los logros alcanzados por los estudiantes relacionados con el tiempo de internalización tanto de los conceptos matemáticos como su utilización en las aplicaciones tecnológicas. Otro aspecto asociado a la internalización de la herramienta es el haber descubierto la potencialidad de la misma.

Tabla 2: Pregunta extraída de la encuesta áulica.

\begin{tabular}{|l|c|c|}
\hline ¿Después de realizada esta experiencia, considera que logró & Año & Año \\
vincular la aplicación de los contenidos de Matemática con la & 2006 & 2007 \\
\cline { 2 - 3 } aplicación al caso de estudio?: & $\%$ & $\%$ \\
\hline 1 Nada & 0 & 0 \\
2 Algo & 47 & 80 \\
3 Mucha & 53 & 20 \\
\hline
\end{tabular}
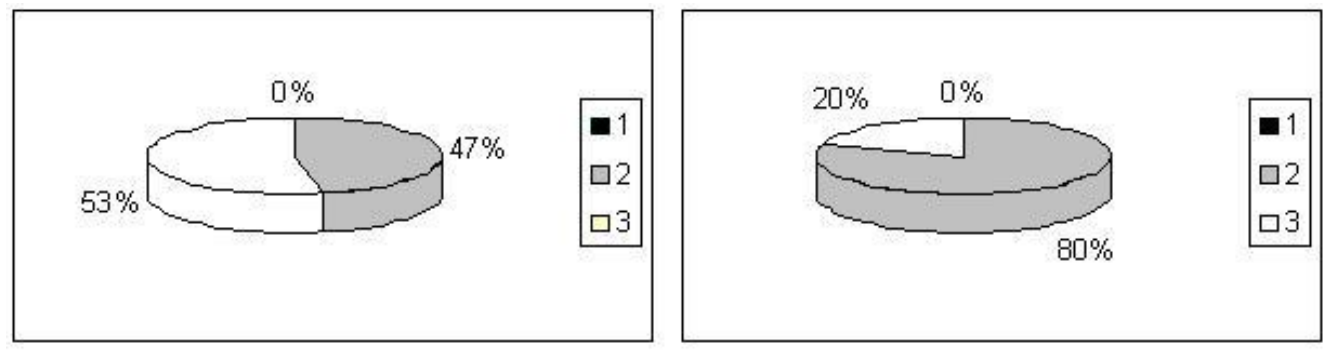

Fig. 3: Lograron vincular la aplicación de los contenidos de Matemática con la aplicación en estudio - Años 2006 y 2007

Tabla 3: Pregunta extraída de la encuesta áulica.

\begin{tabular}{|l|c|c|}
\hline Habiendo estudiado con anterioridad los circuitos eléctricos, con & Año & Año \\
esta experiencia logró interpretar mejor el resultado de la & 2006 & 2007 \\
\cline { 2 - 3 } intensidad de corriente. & $\%$ & $\%$ \\
\hline 1 En lo absoluto & 12 & 22 \\
2 Comprendí más & 57 & 45 \\
3 Totalmente & 31 & 33 \\
\hline
\end{tabular}
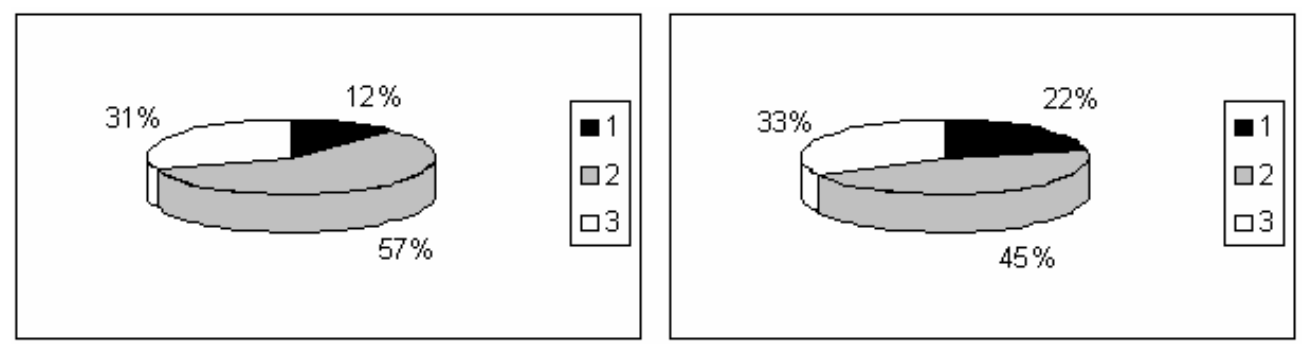

Fig. 4: Lograron interpretar mejor el resultado de la intensidad de corriente - Años 2006 y 2007

Para aquellos estudiantes cuyos conocimientos sobre circuitos eléctricos no eran desconocidos, al evaluar si la experiencia les permitió una mejor interpretación de los resultados un mínimo porcentaje (entre el 12 y el 22\%) indican que en lo absoluto pudieron hacerlo, mientras que alrededor del $50 \%$ han comprendido más y un 30\% indican que totalmente lo lograron (Tabla 3 ) y quienes habían trabajado anteriormente con el software de simulación lograron interpretar mejor los resultados analíticos algo más (65\%) y ciertamente (30\%) (Tabla 4). Los valores obtenidos, permiten inferir la importancia de los conocimientos previos que conjuntamente con la herramienta de simulación posibilitaron una mejor interpretación de los conocimientos adquiridos. Es de resaltar que comienza a generarse una interrelación entre distintas asignaturas (de los primeros años de la carrera) vinculando las Ciencias Básicas con Tecnológicas Básicas, como así la 
incorporación de contenidos (como los relacionados con diagrama de bloques y simulación) que utilizarán a lo largo de la carrera fundamentalmente en Teoría de Control I.

Tabla 4: Pregunta extraída de la encuesta áulica.

\begin{tabular}{|l|c|c|}
\hline ¿Habiendo trabajado con anterioridad con el software de & Año & Año \\
simulación, con esta experiencia logró interpretar mejor los & 2006 & 2007 \\
\cline { 2 - 3 } resultados analíticos hallados manualmente?. & $\%$ & $\%$ \\
\hline 1 Para nada & 0 & 10 \\
2 Algo más & 65 & 60 \\
3 Ciertamente & 35 & 30 \\
\hline
\end{tabular}
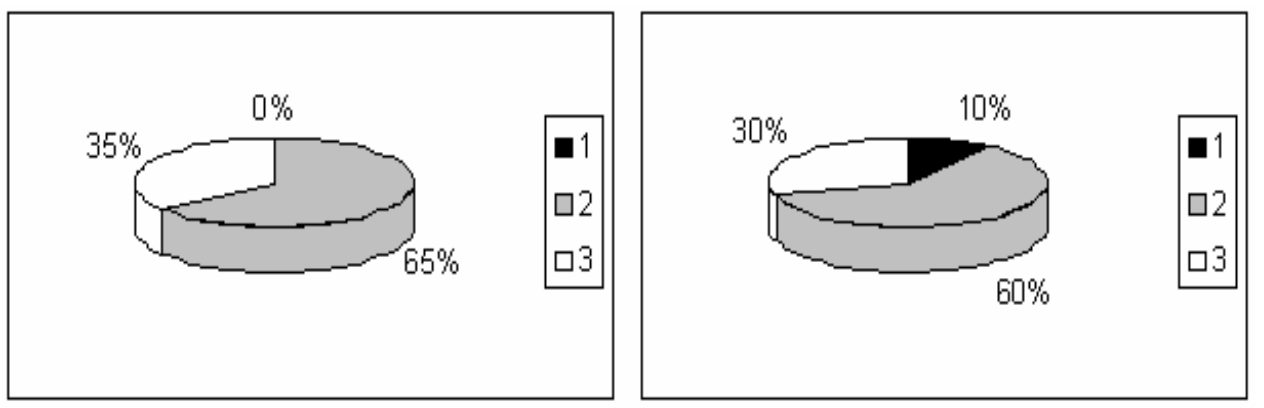

Fig. 5: Lograron interpretar mejor los resultados analíticos - Años 2006 y 2007

Tabla 5: Pregunta extraída de la encuesta áulica.

\begin{tabular}{|l|c|c|}
\hline \multirow{2}{*}{$\begin{array}{l}\text { De acuerdo a la experiencia realizada, opina que la misma } \\
\text { debería repetirse en: }\end{array}$} & $\begin{array}{c}\text { Año } \\
2006\end{array}$ & $\begin{array}{c}\text { Año } \\
2007\end{array}$ \\
\cline { 2 - 3 } & $\%$ & $\%$ \\
\hline $\begin{array}{l}\text { 1 Otros temas de Electrotecnia vinculados con Transformada de } \\
\text { Laplace }\end{array}$ & 19 & 8 \\
$\begin{array}{l}\text { 2 Temas de otras Asignaturas vinculados con Transformada de } \\
\text { Laplace }\end{array}$ & 38 & 42 \\
3 Integrando otros contenidos matemáticos con contenidos de & & 43 \\
otras asignaturas & 50 \\
4 No debería repetirse & 0 & 0 \\
\hline
\end{tabular}
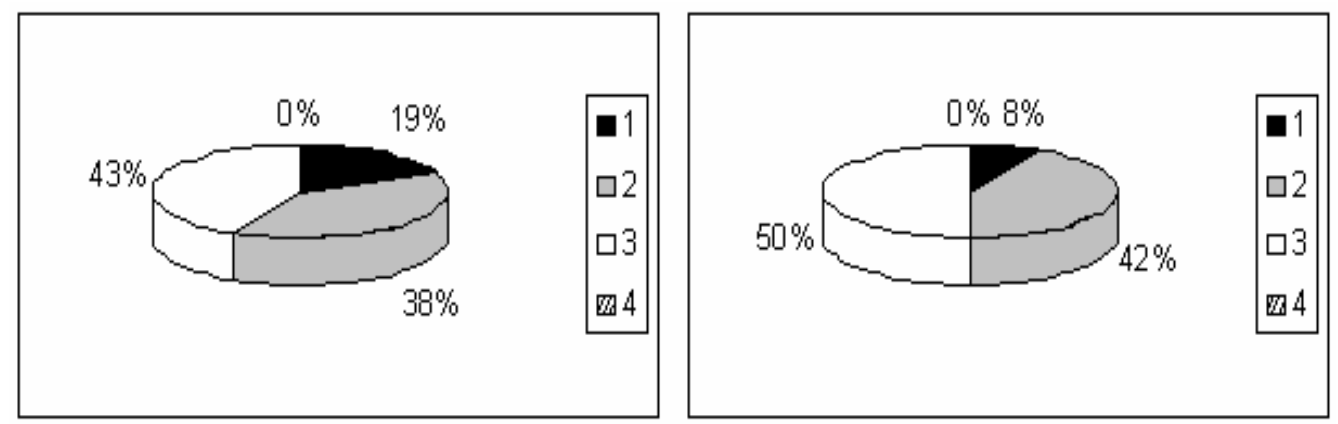

Fig. 6: Opinión si la experiencia debería repetirse - Años 2006 y 2007

Los datos analizados anteriormente focalizan su interés en aspectos propios de la actividad mientras que la interpretación de la Tabla 5 permite evaluar la posibilidad de transferir la propuesta a otras actividades que vinculen temas matemáticos con áreas tecnológicas. Los resultados dejan en claro que no solo se debería ampliar el tema de estudio dentro de la asignatura sino que existe gran interés para que la misma se traslade a otras. Es de destacar que ningún estudiante indica que la experiencia no debería repetirse.

Con lo expuesto se demuestra en mayor o menor medida el grado de alcance de los objetivos propuestos, verificando que los conocimientos matemáticos sirven para resolver problemas tecnológicos, internalizando las herramientas matemáticas. Se ha logrado aunque parcial una interrelación vertical entre estas asignaturas, y a su vez el uso de las herramientas virtuales de simulación permitió una mejor interpretación de los resultados obtenidos analíticamente. 


\section{CONCLUSIONES}

De los indicadores cuantificables obtenidos en las encuestas se concluye que los estudiantes lograron una mejor interpretación de la aplicación de la Transformada de Laplace a situaciones específicas, que la simulación los ayudó a elaborar el análisis de los resultados obtenidos, y el interés en que la propuesta involucre otras asignaturas y contenidos matemáticos.

El entusiasmo y la motivación puesta de manifiesto por los estudiantes al desarrollar las actividades en el aula (que surge a través de indicadores cualitativos de la observación desde la propia experiencia de los docentes) permiten concluir que el cambio propuesto es un disparador en el proceso de enseñanza-aprendizaje.

Las conclusiones, aunque modestas, indican un posible camino de integración de contenidos y una manera de llevarlos a cabo. La experiencia realizada concuerda con la opinión de (Vega Miche, 2003) donde menciona: "A modo de conclusión se puede significar que aunque la estructura disciplinar provoca una fragmentación o parcelación de los contenidos, la integración de conocimientos puede lograrse a partir de las tareas que el profesor diseñe y del modo en que éstas sean abordadas en el proceso de enseñanza aprendizaje".

\section{REFERENCIAS}

Cid Cid, S.; El Uso de Estrategias de Aprendizajes y su Correlación con la Motivación de logro en los Estudiantes, Revista Iberoamericana sobre Calida, Eficacia y Cambio en Educación (REICE), ISSN 16969-4713 (en línea), 6, 3 (2008) http://rinace.net/arts/vol6num3/art4.pdf

Cistac, G., R. Bongianino y F. Kovac; Integrando Conceptos Circuitos RC, Registro Propiedad Intelectual N 583016 (2007), (CD).

Cistac, G., R. Bongianino, J.L. Filippi y F. Kovac; Integrando Conceptos Circuitos RL, Registro Propiedad Intelectual $N^{\circ} 677514\left(2008^{\mathrm{a}}\right)$, (CD)

Cistac, G., R. Bongianino, J.L. Filippi y F. Kovac; Integrando Conceptos Circuitos RCAutoevaluación, Registro Propiedad Intelectual Nº 697501 (2008b), (CD).

Douglas De La Peña, C., G. Bernaza Rodríguez y R. Corral Ruso; Una propuesta didáctica para el aprendizaje de la Física, Revista Iberoamericana de Educación, ISSN 1681-5653 (en línea), 37, 5 (2006), http://www.rieoei.org/experiencias110.htm

Gimeno Sacristán, J. y A. Pérez Gómez; Enseñanza para la comprensión. Comprender y ransformar la enseñanza, Ediciones Morata, S. L, pp. 78-114.Madrid España (1997)

Linn, C. y otros cuatro autores; Teaching and Assessing Knowledge Integration in Science, Sci., ISSN 1095-9203 (en línea), 313 (2006), http://telscenter.org/LinnLeeTinkHusicChui2006.pdf.

Mora, A.; Una experiencia pedagógica en la enseñanza de la matemática sustentada en el enfoque constructivista, Acción Pedagógica, ISSN 1315-401X (en línea), 14, 1 (2005), http://dialnet.unirioja.es/ servelt/articulo?codigo=2969721

Nieto S.; Resolución de Problemas, Matemática Y Computación, Rev. Venezolana de Inform., Tecnol. y Conocimiento, ISSN 1690-7515 (en línea), 2, 2 (2005), http://www.dialnet.unirioja.es/ servelt/articulo?codigo $=1985688$

Ojeda, M. y M .Alcalá; La enseñanza en las aulas universitarias. Una mirada desde las cátedras: aspectos curriculares que inciden en las prácticas pedagógicas de los equipos docentes, Revista Iberoamericana de Educación, ISSN 1681-5653 (en línea), 34, 1, 25 de octubre (2007), http://www.rieoei.org/inv edu35.htm

Ruiz Socarras, J.; Problemas actuales de la enseñanza aprendizaje de la matemática., Revista Iberoam. de Educación, ISSN 1681-5653 (en línea), 47, 3, (2008), http://www.rieoei.org/2359.htm 
Santos Trigo, L.; La transferencia del conocimiento y la formulación o rediseño de problemas en el aprendizaje de las matemáticas, Revista mexicana de investigación educativa, ISSN 1405-6666, 2, 3 (en línea), (1997), http://dialnet.unirioja.es/servlet/artículo?codigo=300359

Santos Trigo, M.; La Resolución de Problemas Matemáticos: Avances y Perspectiva en la Construcción de una Agenda de Investigación y Práctica, Investigación en Educación Matemática XII, ISBN 978-84-934488-9-9 (en línea) (2008), http://www.seiem.es/publicaciones/archivospublicaciones/ actas/Actas12SEIEM/Seminario2SantosTrigo.pdf

Valdés Castro, P. y R. Valdés Castro; Características del proceso de enseñanza-aprendizaje de la física en las condiciones contemporáneas., Revista de Investigación y experiencias didácticas, ISSN 0212-4521 (en línea), 17, 3 (1999), http://dialnet.unirioja.es/servlet/oaiart?codigo=94977

Vásquez, S., P. Bustos, G. Núñez y C. Mazzitelli; Planteo de situaciones problemáticas como estrategia integradora en la enseñanza de las ciencias y la tecnología. Enseñanza de las Ciencias, ISSN (en 1579-1513 línea), http://www.saum.uvigo.es/reec/volumenes3/Numero1/Art4.pdf

Vega Miche, R.; La integración de los contenidos: Un reto para un plan de estudios disciplinar, Rev. Superior Cubana, ISSN 0257-4314 (en línea), 23, 3 (2003), http://www.dic.uh.cu/Revistas/Educ Sup/ 0302002/Art070303.pdf 